\title{
Mesh-related complications of laparoscopic lateral suspension
}

\begin{abstract}
Objective: Restorative procedures are essential for sexually active vaginal vault prolapse (VVP) cases. There are concerns about the long learning curve and major complications of sacrocolpopexy. Laparoscopic lateral suspension has a relatively short learning curve and no reported major complications. However, there are concerns about the use of mesh in prolapse surgeries and limited data is available about mesh complications with laparoscopic lateral suspension. This study aimed to establish the mesh-related complications after laparoscopic lateral suspension.
\end{abstract}

Study Design: We carried out a retrospective analysis of patients who underwent laparoscopic lateral suspension for symptomatic VVP at three tertiary hospitals. Cases were identified from theatre records with coding data and the universities database. Demographic features, concomitant procedures, duration of surgery, intra-operative complications, hospital stay, change in pelvic organ prolapse quantification point $\mathrm{C}$, Patient Global Impression of Improvement scale records, mesh-related complications and their management are presented.

Results: A total of 120 patients underwent laparoscopic lateral suspension for symptomatic vault prolapse between 2014-2019. In total, mesh complications developed in 3 women (2.5\%). While two vaginal mesh exposures were successfully managed with topical estrogen, one required surgical excision. One lateral mesh arm was loosened from the skin causing pain and itching. From 110 Patient Global Impression of Improvement scores, 106 women $(96 \%)$ ) described their prolapse as 'very much' or 'much' better, 4 patients $(3.6 \%)$ reported 'no change' and no one reported worsening of symptoms.

Conclusions: This series suggests that laparoscopic lateral suspension has low risk of mesh-related complications. It is a safe and effective procedure for symptomatic vault prolapse with high rates of patient satisfaction.

Keywords: pelvic organ prolapse, mesh complications, laparoscopic lateral suspension, mesh
Volume II Issue 4 - 2020

\section{Adeviye Elçi Atılgan, Șükriye Leyla Altuntaș \\ Department of Obstetry and Gynecology, Faculty of Medicine, Istanbul Medipol Üniversity, İstanbul-Bağcılar,Turkey}

Correspondence: Adeviye Elçi Atılgan ,Asistant Professor MD Department of Obstetry and Gynecology, Faculty of Medicine, Istanbul Medipol Üniversity, İstanbul-Bağcılar,Turkey, Tel +90505 75537 83, Email dradeviye@gmail.com

Received: May 27, 2020 | Published: July 202020

\section{Introduction}

Descent of the apical compartment of the vagina after hysterectomy is known as vaginal vault prolapse (VVP) or cuff prolapse and it has a cumulative incidence of $0.5 \% .{ }^{1}$ VVP can be corrected with various surgical techniques via vaginal, abdominal or laparoscopic routes. ${ }^{2}$ Among them laparoscopic sacrocolpopexy is widely preferred with reported objective success rates up to $92 \%$ and decreased hospital stay and blood loss compared to the abdominal route. ${ }^{3}$ However, vascular, ureteral or neurological injury that may be encountered during dissection of the promontorium are rare but serious complications of the technique. ${ }^{4}$ Laparoscopic lateral suspension (LLS) is a welldefined method as an alternative to sacrocolpopexy for correction of apical prolapse, avoiding promontorium dissection. ${ }^{5}$ Mesh-related complications after LLS present similar to other pelvic floor surgeries performed with mesh. However, there is limited data about mesh complications after LLS in the literature.

In this study, we aimed to share mesh-related complications after LLS performed for symptomatic VVP by coding them according to the joint International Urogynecology Association (IUGA)/International Continence Society (ICS) mesh-related complication classification system. ${ }^{6}$

\section{Materials and methods}

\section{Patient selection}

This is a retrospective analysis of patients who underwent LLS for symptomatic stage $>2$ posthysterectomy vaginal vault prolapse according to the Pelvic Organ Prolapse Quantification system (POP-Q) in the period from April 2014 to February 2019 at three tertiary hospitals.

\section{Surgical technique}

LLS was performed as described originally for vault prolapse by Dubuisson et al. ${ }^{5}$ Under laparoscopic view, a vaginal probe was inserted through the vagina and precut cross-shaped mesh with two long arms of type 1 lightweight, polypropylene, microporous, monofilament mesh was sutured with nonabsorbable 0-polypropylene suture (Prolene; Ethicon/Johnson \& Johnson) over the anterior and posterior vaginal wall after dissection of vesicovaginal and rectovaginal spaces. If concomitant cystocele was present, the anterior arm of the mesh was placed under the bladder. If concomitant rectocele was present, the posterior arm of the mesh was extended up to the perineal body. The two long lateral arms were pulled under the peritoneum with an endoscopic grasper up to the posterior and anterior superior iliac spine. After tension-free vaginal vault suspension was 
performed, each mesh arm was cut at the level of the skin. The lateral distal part of the mesh arms were not sutured to the abdominal fascia. Then, mesh was completely retroperitonealized with absorbable 2-0 polyglactin suture (Monocryl; Ethicon/Johnson \& Johnson).

Vaginal examination was performed at the end of each operation to control cuff level and make sure there was no prolene suture exposure.

\section{Follow-up/assessment of prolapse}

Patients were examined regularly at 1 month and 6 months later and yearly by their surgeons. Prolapse was reevaluated at left lateral position using the Valsalva maneuver in each control examination period. Post-operative changes of point $\mathrm{C}$ or de nova prolapsed compartment points, if any, were determined by using the Pelvic Organ Prolapse Quantification (POP-Q) system. During the followup period, patients were also asked to assess success of their surgery using the Patient Global Impression of Improvement Scale (PGI-I). This is a translated questionnaire scale that asks patients to rate their current condition compared with before on a scale from 1 (very much better) to 7 (very much worse). ${ }^{7}$

\section{Data collection}

Ethics committee approval was not obtained because it was a retrospective study, but an approval form was obtained from the universities' publication audit committees. Cases were collected from database and coding departments of two university hospitals using the terms 'Laparoscopic Lateral Suspension' and 'Vaginal Vault Prolapse'. Patient demographic data of age, parity, menopause status, body mass index, previous operation histories, concomitant procedures, intra-operative and post-operative complications, duration of surgery, amount of blood loss, and preoperative and post-operative change in position of point $\mathrm{C}$ according to POP-Q system were documented retrospectively from hospital records. Patients who represented with complaints such as dyspareunia, pelvic pain, postcoital bleeding, recurrent vaginal discharge, hematuria, and fever which may be associated with mesh complications, management of these complications or who needed a further operation were identified from the file records until the data was collected (February 2019). Detected mesh-related complications were coded individually according to the joint International Urogynecological Association (IUGA)/ International Continence Society (ICS) mesh-related complication classification calculator. $^{5}$

In order to detect mesh-related complications after only LLS, concomitant midurethral sling procedures, history of previously pelvic floor surgery with mesh or performed concomitant anterior/ posterior intravaginal slingplasty (IVS) were excluded from the study.

\section{Analysis}

The primary outcome of this study was the incidence of meshrelated short-term or long-term complications. Secondary outcomes were duration of surgery, length of hospital stay, change in point $\mathrm{C}$, concomitant procedures, further performed procedures for prolapse during the follow up period and patients' satisfaction score. The variables associated with mesh-related complications were assessed by using multivariate analysis.

\section{Results}

A total of 132 cases who underwent LLS were identified from the universities' databases and coding departments between April
2014 and February 2019. Eleven were excluded from the study because of midurethral sling procedures (8-TVT, 3-TOT) for stress urinary incontinence in the same session. One patient who had sacrocolpopexy performed for vault prolapse and 2 months later LLS performed in our clinic for recurrence was excluded from the study too. So, the study was conducted with 120 patients. As shown by the patient characteristics in Table 1, mean age of patients was 61 years (range 41-81), mean parity was 2 (range $0-5$ ) and mean BMI was 28.6 (range 22-51) (Table 1).

Table I Patient Characteristics

\begin{tabular}{ll}
\hline Characteristics & Mean (range) \\
\hline Age $(\mathrm{I} 20)$ & $61.2(33-8 \mathrm{I})$ \\
$\mathrm{BMI}\left(\mathrm{kg} / \mathrm{m}^{2}\right)(\mathrm{I} 20)$ & $28.6(22-5 \mathrm{I})$ \\
Point C pre-operatively $(\mathrm{II})$ & $+2 . \mathrm{I}(-6$ to $+\mathrm{I})$ \\
Point C post-operatively $(\mathrm{II})$ & $-7.4(-3$ to -10$)$ \\
\hline
\end{tabular}

BMI, body mass index

A total of 24 patients $(20 \%)$ underwent concomitant procedures with LLS (Table 2): 15 patients had perineoplasty for perineal insufficiency; 8 patients had laparoscopic Burch colposuspension; 2 patients had ovarian cystectomy; 3 patients underwent adhesiolysis and 4 patients underwent laparoscopic paravaginal repair. Of the patients, 118 were sexually active. The mean time of surgery was 184min (range 90-245). The mean hospital stay was 2.2 days (range $2-8$ ). The median time from surgery was 73 months (6years, 1 month).

Table 2 Concomitant surgery with LLS

\begin{tabular}{lc}
\hline Concomitant surgery & N (\%) \\
\hline Perineoplasty & $15(12.5)$ \\
Burch & $8(6.6)$ \\
Ovarian cystectomy & $2(1.6)$ \\
Paravaginal Repair & $4(3.3)$ \\
Adhesiolysis & $3(2.5)$ \\
\hline
\end{tabular}

All the patients attended routine follow-up assessment. A total of 118 women had documented POP-Q scoring of point C preoperatively. The mean value was +2.1 (range; -6 to +12 ) (Table 1). Post-operatively, 113 women had documented point $C$ with a mean value of -7.4 (range; -3 to -10) (Table 1). In 112 cases, both pre- and post-operatively point $\mathrm{C}$ value was documented. The mean change in point $\mathrm{C}$ was -7.5 (median -7 , range 0 to-12). At 6 th month routine control examination, stage 1 de nova enterocele was detected according to POP-Q system. The patient was asymptomatic and records revealed that we performed LLS and concomitant laparoscopic Burch procedure for stress urinary incontinence.

Patient Global Impression of Improvement (PGI-I) scores were available for 110 patients. A total of 106 women (96\%) described their prolapse as 'very much' or 'much' better after LLS, 4 patients (3.6\%) reported 'no change' and no one reported worsening of symptoms. 


\section{Mesh-related complications}

There was no recorded intraoperative mesh-related complication. In 120 patients, 3 patients $(2.5 \%)$ were found to have mesh-related complications. We collected each complication by coding based on international standard category $(\mathrm{C})$, time $(\mathrm{T})$ and site $(\mathrm{S})$ classes and divisions with the joint IUGA and ICS classification of the complications related directly to the insertion of prostheses (meshes, implants, tapes) and grafts in female pelvic floor surgery ${ }^{6}$

a. A 49-year old patient had undergone laparoscopic lateral suspension for POP-Q stage 3 vaginal vault prolapse and perineorrhaphy for perineal insufficiency. Fourteen months later, she complained of pain during sexual intercourse. On physical examination, mesh exposure of $6 \mathrm{~mm}$ by $8 \mathrm{~mm}$ was detected at the apex of the vaginal cuff. She was successfully treated with topical estrogen for 5 months ( $2 B c T 4 S 1)$.

b. A 51-year-old woman reported recurrent vaginal discharge and febrile at $38 \mathrm{C}$ starting 9 months after LLS. A $20 \times 15 \mathrm{~mm}$ mesh extrusion was revealed in the anterior vaginal wall. After excision of the mesh under local anesthesia with oral antibiotics, she had not further problems (3C T3 S1).

c. A 48-year old patient complained of pain and itching at left lateral mesh suspension skin site during physical activities starting 1 month after LLS operation. While palpating the left side mesh fiber lateral site, there was pain with tenderness but no epithelial separation. She was successfully managed by loosening left lateral site terminal mesh from surrounding tissue under skin and skin was primary re-sutured under local anesthesia with oral antibiotics and analgesic (1Bd T2 S4).

\section{Discussion}

Vaginal vault prolapse affects $1 \%$ of women who undergo hysterectomy all around the world. ${ }^{8}$ There is no evidence about the efficacy of pelvic floor exercises. ${ }^{9}$ Pesser insertion to the vaginal cuff is a short term management method for VVP. ${ }^{10}$ Pesseries are not very suitable especially for sexually active women under fifty years old. Therefore, a surgical approach for VVP becomes inevitable. Sacrocolpopexy, especially the laparoscopic approach, provides high success and low recurrence rates. ${ }^{11}$ It has been widely performed since 1957 for apical pelvic organ prolapse. ${ }^{11-13}$ So, there are quite a lot of data, and well-documented publications about short-term and long-term complications after SCP in the literature from 1957 to the present.

At present, synthetic meshes insertion doesn't allowed to by the transvaginal route due to increased number of mesh related complications. ${ }^{13,14}$ However,there is no any forbidden to using by laparoscopic, robotic or abdominal routes for pelvic organ prolapse surgeries. In parallel with this, there can be reveal different type and site of mesh-associated complications. ${ }^{14}$ Although rare, these complications may have serious consequences. ${ }^{15}$ In a review of complications defined as serious after SCP, all of them were reoperated on under general anesthesia and more than one third of them $(37.0 \%)$ needed several reoperations. ${ }^{15}$ LLS has been performed since 2013 when it was first published as an alternative to SCP. ${ }^{5}$ However, physicians dealing with pelvic floor surgery need more detailed standardized data about mesh complications with this technique. Physicians' lack of knowledge and experience may cause further severe morbidity if complications are not diagnosed and treated appropriately.

The most common complication associated with using mesh is mesh exposure and erosion. Although symptom presentation time may vary from days to years; recurrent vaginal discharge, intermittent bleeding, hematuria, pain during sexual intercourse (dyspareunia) or partner pain (hyspareunia) are common symptoms of mesh exposures. ${ }^{16}$ The mesh erosion rate after LLS was reported as $3.4 \%$ to $5.5 \%$ in many publications. ${ }^{17-19}$ Indeed, there was no reported intra-operative or post-operative life-threatening mesh-related complications with LLS up to now. Mereu et al. reported only one postoperative Clavien-Dindo grade 3 complication in their 119 cases $(0.8 \%) .{ }^{20}$ Veit-Rubin et al. published grade 3 or higher Clavien-Dindo complication rate as $2.2 \%$ in 417 patients with LLS performed for pelvic organ prolapse. ${ }^{18}$ On the other hand, physicians may have to manage a challenging scenario any time after LLS. Milani et al. reported that they had to perform laparoscopic subtotal mesh excision from bladder, concomitant hysterectomy and bladder repair for pelvic pain, dyspareunia and bladder erosion 2 years after LLS. ${ }^{21}$

Almost all mesh-related complications were defined as "mesh erosion" by authors. However, this term doesn't completely correspond to the specific mesh-related complication or patient's clinic whereas, the term "erosion" is not included in the IUGA-ICS terminology document. ${ }^{6}$ The American Urogynecologic Society and International Urogynecological Association also recommend using the 'mesh exposure' term with the anatomic location of mesh exposure. ${ }^{22}$

Although it is not a direct mesh-related complication, suture erosion is an important complication that may cause discharge, pain, bleeding, and ascendant infection. ${ }^{23}$ We routinely use prolene sutures and we didn't encounter any suture erosion case during our follow-up period. Georgina at al. found a strong association with an increased risk of suture erosion with Ethibond sutures (OR 10.82 95\%CI 2.54$46.10 \mathrm{p}=0.001$; comparative use of PDS suture). ${ }^{24}$ We also routinely checked the vagina when each suture was made during surgery. If we had passed any transfix suture over the vagina while attaching the mesh, we removed the suture at that time because it is well documented that transfixing sutures increases the risk of exposure. ${ }^{23,24}$ In addition it will cause recurrent vaginal discharge. It may disturb couple and negatively effect sexual activity.

\section{Conclusion}

In this article, we report data and experience from three tertiary units about mesh-related complications after laparoscopic lateral suspension for posthysterectomy vaginal vault prolapse. The study found that laparoscopic lateral suspension is an effective and safe technique with few mesh-related complications, similar to other published studies.

\section{Acknowledgments}

We thank Associate Prof. Dr. İsa Aykut Özdemir for giving permission to share patients data operated with LLS technique at Bakırköy Sadi Konuk Research Hospital.

\section{Funding}

None. 


\section{Conflicts of interest}

All authors declare that they have no conflicts of interest in regard to this study.

\section{References}

1. Dällenbach $P$, Kaelin-Gambirasio I, Jacob S, et al. Incidence rate and risk factors for vaginal vault prolapse repair after hysterectomy. Int Urogynecol J Pelvic Floor Dysfunct. 2008;19(12):1623-1629.

2. Uzoma A, Farag KA. Vaginal vault prolapse. Obstet Gynecol Int. 2009;2009:275621

3. Ganatra AM, Rozet F, Sanchez-Salas R, et al. The current status of laparoscopic sacrocolpopexy:a review. Eur Urol. 2009;55(5):1089-1103.

4. Barber MD, Maher C. Apical prolapse. Int Urogynecol J. 2013;24(11):1815-1833.

5. Dubuisson J, Eperon I, Dällenbach P, et al. Laparoscopic repair of vaginal vault prolapse by lateral suspension with mesh. Arch Gynecol Obstet. 2013;287(2):307-312.

6. Haylen BT, Freeman RM, Swift SE, et al. An International urogynecological association (IUGA)/International continence society (ICS) joint terminology and classification of the complications related directly to the insertion of prostheses (meshes, implants, tapes) and grafts in female pelvic floor surgery. Neurourol Urodyn. 2011;30(1):2-12.

7. Srikrishna S, Robinson D, Cardozo L. Validation of the patient global impression of improvement (PGI-I) for urogenital prolapse. Int Urogynecol J. 2010;21(5):523-528.

8. Dubuisson J, Veit-Rubin N, Bouquet de Jolinière J, et al. Laparoscopic lateral suspension: benefits of a cross-shaped mesh to treat difficult vaginal vault prolapse. J Minim Invasive Gynecol. 2016;23(5):672.

9. Lamers BH, Broekman BM, Milani AL. Pessary treatment for pelvic organ prolapse and health-related quality of life: a review. Int Urogynecol J. 2011;22(6):637-644.

10. Jia X, Glazener C, Mowatt G, et al. Systematic review of the efficacy and safety of using mesh in surgery for uterine or vaginal vault prolapse. Int Urogynecol J. 2010;21(11):1413-1431.

11. Nygaard IE, McCreery R, Brubaker L, et al. Abdominal sacrocolpopexy: a comprehensive review. Obstet Gynecol. 2004;104(4):805-823.

12. Ameline A, Huguer J. Posterior suspension to the lumbo-sacral disk; abdominal method of replacement of the utero-sacral ligaments. Gynecol Obstet (Paris). 1957;56(1):94-98.
13. Arthure HG, Savage D. Uterine prolapse and prolapse of the vaginal vault treated by sacral hysteropexy. J Obstet Gynaecol $\mathrm{Br}$ Emp. 1957;64(3):355-360.

14. Kasyan G, Abramyan K, Popov AA, et al. Mesh-related and intraoperative complications of pelvic organ prolapse repair. Cent European $J$ Urol. 2014;67(3):296-301.

15. Arsene E, Giraudet G, Lucot JP, et al. Sacral colpopexy: long-term mesh complications requiring reoperation(s). Int Urogynecol J. 2015;26(3):353-358.

16. Ganer Herman H, Raz N, Gold E, et al. Risk of mesh erosion after pelvic organ prolapse repair with or without concomitant vaginal hysterectomy. Isr Med Assoc J. 2019;21(6):399-403.

17. Eperon I, Luyet C, Yaron M, et al. Laparoscopic management of genital prolapse by lateral suspension using mesh: a series of 377 patients. Rev Med Suisse. 2011;7(314):2084-2088.

18. Veit-Rubin N, Dubuisson JB, Gayet-Ageron A, et al. Patient satisfaction after laparoscopic lateral suspension with mesh for pelvic organ prolapse: outcome report of a continuous series of 417 patients. Int Urogynecol J. 2017;28(11):1685-1693.

19. Dällenbach P, De Oliveira SS, Marras S, et al. Incidence and risk factors for mesh erosion after laparoscopic repair of pelvic organ prolapse by lateral suspension with mesh. Int Urogynecol J. 2016;27(9):1347-1355.

20. Mereu L, Tateo S, D'Alterio MN, et al. Laparoscopic lateral suspension with mesh for apical and anterior pelvic organ prolapse: A prospective double center study. Eur J Obstet Gynecol Reprod Biol. 2020;244:16-20.

21. Milani R, Manodoro S, Passoni P, et al. Surgical management of bladder erosion and pelvic pain after laparoscopic lateral suspension for pelvic organ prolapse. Int Urogynecol J. 2020;31(4):843-845.

22. Developed by the Joint Writing Group of the American Urogynecologic Society and the International Urogynecological Association. Joint position statement on the management of mesh-related complications for the FPMRS specialist. Int Urogynecol J. 2020;31(4):679-694.

23. Baines $\mathrm{G}$, Price N, Jefferis $\mathrm{H}$, et al. Mesh-related complications of laparoscopic sacrocolpopexy. Int Urogynecol J. 2019;30(9):1475-1481.

24. Freeman RM, Pantazis K, Thomson A, et al. A randomised controlled trial of abdominal versus laparoscopic sacrocolpopexy for the treatment of post-hysterectomy vaginal vault prolapse: LAS study. Int Urogynecol J. 2013;24(3):377-384. 